\title{
High Prevalence of Low Serum Paraoxonase-1 in Subjects with Coronary Artery Disease
}

\author{
Narayani Jayakumari* and Gopalan Thejaseebai \\ Division of Biochemistry, Sree Chitra Tirunal Institute for Medical Sciences and Technology, \\ Thiruvananthapuram-695011, Kerala, India
}

Received 14 November, 2008; Accepted 5 February, 2009

\begin{abstract}
Summary Paraoxonase-1 (PON-1), an HDL-associated enzyme has been shown to possess antioxidant/anti-inflammatory properties and protect against atherogenesis. The aim of the study was to explore the association of PON-1, with lipids, antioxidant-vitamins and lifestyle in male subjects with and without angiographically documented coronary artery disease (CAD) and in healthy volunteers. PON-1 activity was measured spectrophotometrically using phenyl acetate as substrate. PON-1 and HDL-Cholesterol were significantly lower and lipid peroxides and triglycerides were higher in CAD patients than in normal coronary and control subjects. PON-1 activity showed positive association with HDL-C and inverse relation with lipid peroxides and no association with antioxidant vitamins in healthy subjects. Smoking habit, alcohol intake and diabetes mellitus did not seem to influence PON-1 activity. Serum levels of PON-1 and HDL-C showed inverse association with the presence of CAD but are not related to the severity of disease in terms of number of diseased vessels. Interventional means by diet/drugs to enhance PON-1 activity may contribute to attenuation of atherosclerosis.
\end{abstract}

\section{Key Words: Atherosclerosis, Paraoxonase, HDL, Oxidativestress}

\section{Introduction}

Atherosclerosis, the fundamental cause of majority of heart attacks, is a disease of chronic inflammation and lipid accumulation. Although the precise mechanisms involved in coronary artery disease (CAD) are not clear, current theory predicts that it is caused by an inflammatory response of arterial wall macrophages to oxidized low-density lipoprotein (LDL) $[1,2]$. Oxidative modification of LDL in the artery wall is now widely regarded as playing an important role in the initiation and progression of atherosclerosis. High-density lipoprotein (HDL), on the other hand, normally plays an anti-atherogenic role. The protective capacity of HDL has been ascribed primarily to its ability to remove

\footnotetext{
*To whom correspondence should be addressed. Tel: +91-471-2524178 Fax: +91-471-2446433

E-mail: jaya@sctimst.ac.in
}

excess cholesterol from peripheral tissues in the reverse cholesterol transport pathway. Recent studies have suggested more diverse mechanisms. HDL inhibits LDLoxidation $[3,4]$ and this may contribute to inverse association between plasma HDL levels and risk of developing CAD. These protective effects of HDL have been attributed to the various proteins associated with HDL. Paraoxonase-1 (PON-1) is one such protein that had been reported to possess antioxidant/anti-inflammatory properties [5].

PON-1, an aryldialkylphosphatase EC 3.1.8.1, is an ester hydrolase associated with HDL in blood [6]. It is well established that human serum PON-1 catalyzes the hydrolysis of toxic organophosphate insecticides, nerve gases, and aromatic carboxylic acid esters and oxidized phospholipids. The hydrolysis of oxidized phospholipids by PON-1 destroys the biologically active lipids in oxidized LDL. This action of PON-1 could delay the development of atherosclerosis. There is growing evidence that reduced activity of HDLassociated PON-1 is predictive of vascular disease in humans 
[2, 7-9] including results from prospective studies [10]. A role of PON-1 in vascular disease is also strongly supported by knockout and transgenic mouse studies [11, 12]. However the precise mechanism by which PON-1 influence the risk of vascular disease is not yet completely known. Its activity is inactivated by oxidized LDL and preserved by antioxidants [7]. Unlike chain-breaking antioxidants, HDL prevents the accumulation of lipid peroxides in LDL and in the vessel wall for several hours, continuing to do so long even after fat-soluble antioxidants have been exhausted and this activity seems to be due to PON-1 [13].

There is an enormous ( $\sim 40$ fold) variation in serum PON-1 activity between individuals due in part, to common polymorphism [14]. A variety of external factors, such as antioxidants, fatty foods, lifestyle etc, can also positively as well as negatively influence the activity of human PON-1. The serum concentration of HDL-cholesterol has long been known to have an inverse association with the development of atherosclerosis that was confirmed by our previous studies conducted on CAD patients $[15,16]$. In addition, enhanced oxidative stress and altered antioxidant enzymes, mainly super oxide dismutase, have been found in CAD patients during stable and unstable anginal syndromes [16]. In view of these, the aims of the present study were to explore the relationship between serum Paraoxonase- 1 and oxidants/antioxidant-vitamins, lipids, lipoproteins and lifestyle habits in male subjects having established CAD and non-CAD as well as to determine the association of PON-1 with the extend of coronary artery disease.

\section{Patients and Methods}

Patients included in this study were the ones admitted in the Cardiology ward of this Institute for coronary angiography as a confirmatory test for coronary artery disease. Male patients from the age of 25-60 years, who were not taking any lipid lowering drugs and vitamin supplementation at the time of admission, were recruited for this study. Patients having other types of heart disease, such as valvular heart disease, cardiomyopathies etc as well as those having renal and hepatic diseases were also excluded. Based on the angiographic reports patients were categorized into two groups as having coronary artery disease [CAD, $n=284]$ and normal coronary [non-CAD, $n=45]$. Details of patients recorded at the time of admission include age, sex, height, weight, smoking habits, and history of diseases-diabetes mellitus, hypertension and medication. All patients were receiving standard drugs-nitrates, $\beta$-blockers and/or calcium channel blockers for the treatment of angina and hypertension. Those having diabetes were receiving oral hypoglycemic drugs. Male healthy volunteers $(n=245)$ from the hospital staff and their relatives who were free of any symptoms of chest pain were included as controls for this study. Lack of CAD was assessed by use of a health questionnaire and all had no history suggestive of CAD. Prior informed written consent was obtained from all subjects for examining blood samples and providing details regarding health and lifestyle habits required for the study as per our Institute's human research ethical guidelines.

\section{Blood sampling}

Venous blood sample were obtained from all subjects in the morning after an overnight fast of $\sim 12 \mathrm{~h}$. Serum was collected by low speed centrifugation and subjected to various biochemical analyses.

\section{Analytical methods}

Serum lipids. Serum total cholesterol and HDL-cholesterol (after centrifugation of apo-B containing lipoproteins) were determined by the recommended CHOD-PAP method using enzyme assay kits (Olympus Life and Material Science Europa GmbH, Germany). Serum triglycerides were measured by the enzymatic GPO-PAP method. LDL-cholesterol was calculated according to the Friedewald's formula [LDL$\mathrm{C}=$ total cholesterol $-($ HDL-C + Triglycerides $/ 5) \mathrm{mg} / \mathrm{dl}]$. The concentration of oxidants as lipid peroxides in serum was quantitated by the thiobarbituric acid method using malondialdehyde as standard [17].

Antioxidant vitamins. Vitamin-E, $\mathrm{C}$ and $\beta$-carotene were quantitated by recommended spectrophotometric and spectrofluorimetric methods [18-20]. Vitamin-E quantification involved saponification of serum in the presence of pyrogallol to prevent destruction of tocopherol by alkali and extraction of tocopherol in hexane. The specific fluorescence of tocopherols in hexane extract was measured at wavelengths, excitation $286 \mathrm{~nm}$ and emission $350 \mathrm{~nm}$, using FP-750 spectrofluorometer (Jasco, Tokyo, Japan). Vitamin-C/ Ascorbic acid determination depended on the reduction of ferric ion to ferrous ion by ascorbic acid and the ferrous ion was determined as the red-orange $\alpha, \alpha$-dipyridyl chromophore at $525 \mathrm{~nm}$ using UV-VIS-1601 spectrophotometer (Shimadzu, Kyoto, Japan). For quantitating $\beta$-carotene, an extract of the serum in petroleum ether was prepared and the absorbance was monitored at $450 \mathrm{~nm}$ against a standard of crystalline $\beta$-carotene using a spectrophotometer.

Paraoxonase-/arylesterase activity. PON-1 activity was measured using phenyl acetate as substrate [21]. Briefly, an aliquot of serum was added to a reaction mixture consisting of $4 \mathrm{mMol} /$ liter phenyl acetate in $50 \mathrm{mMol} /$ liter Tris acetate buffer, pH 7.8, containing $20 \mathrm{mMol} /$ liter calcium chloride. The rate of hydrolysis of phenyl acetate was monitored at $270 \mathrm{~nm}$ in a spectrophotometer using a molar absorption coefficient at $270 \mathrm{~nm}$ of 1310 liter. $\mathrm{Mol}^{-1} \cdot \mathrm{cm}^{-1}$. 
Table 1. Basic Characteristics of the study participants

\begin{tabular}{lccc}
\hline & control & CAD & Normal coronary \\
\hline Male subjects $(n)$ & 245 & 284 & 45 \\
Mean age (yrs) & $44 \pm 10.2$ & $51 \pm 6.9$ & $49 \pm 8.2$ \\
Cigarette smoking (\%) & 17 & 65 & 9 \\
Diabetics Mellitus (\%) & 13 & 39 & 20 \\
Hypertension (\%) & 13 & 41 & 25 \\
Overweight [BMI $\left.\left(\mathrm{Kg} / \mathrm{m}^{2}\right) \geq 25\right](\%)$ & 31 & 50 & 44 \\
\hline
\end{tabular}

Table 2. Serum concentrations of lipids and lipoproteins in subjects with coronary artery disease, normal coronary and controls $($ mean \pm SD.mg\%)

\begin{tabular}{lcccc}
\hline & Total Cholesterol & Triglycerides & LDL-C & HDL-C \\
\hline Control $(n=245)$ & $220 \pm 44$ & $141 \pm 68$ & $149 \pm 38$ & $42 \pm 9.5$ \\
CAD $(n=284)$ & $206 \pm 42^{\mathrm{a}}$ & $164 \pm 72^{\mathrm{a}}$ & $140 \pm 40^{\mathrm{b}}$ & $33 \pm 7.7^{\mathrm{aa}}$ \\
Normal Coronary $(n=45)$ & $204 \pm 59$ & $148 \pm 79$ & $137 \pm 51$ & $38 \pm 9.4^{\mathrm{b}}$ \\
\hline
\end{tabular}

control vs $\mathrm{CAD} \mathrm{a}=p<0.001 \& \mathrm{~b}=p<0.01$; Control vs normal coronary $\mathrm{b}=p<0.01$; CAD vs normal coronary $\mathrm{a}=p<0.001$

Table 3. Paraoxonase-1 activity and oxidant/antioxidant vitamin status in subjects with coronary artery disease, normal coronary and controls (mean $\pm \mathrm{SD}$ )

\begin{tabular}{lccc}
\hline & Control $(n=245)$ & CAD $(n=284)$ & Normal Coronary $(n=45)$ \\
\hline Paraoxonase-1 $(\mathrm{kU} / \mathrm{L})$ & $100 \pm 22$ & $88 \pm 25^{\text {a c }}$ & $96 \pm 22$ \\
Lipid peroxides as Malondialdehydes $(\mathrm{nM} / \mathrm{ml})$ & $1.85 \pm 0.51$ & $2.12 \pm 0.61^{\mathrm{ac}}$ & $1.88 \pm 0.65$ \\
Vitamin E $(\mu \mathrm{g} / \mathrm{dl})$ & $16 \pm 6.5$ & $16 \pm 4.8$ & $17 \pm 5.4$ \\
Vitamin $\mathrm{C}(\mathrm{Mg} / \mathrm{dl})$ & $0.62 \pm 0.29$ & $0.52 \pm 0.27^{\mathrm{a}}$ & $0.55 \pm 0.29$ \\
$\beta$-Carotene $(\mu \mathrm{g} / \mathrm{dl})$ & $102 \pm 34$ & $91 \pm 41^{\mathrm{a}}$ & $92 \pm 36$
\end{tabular}

control vs $\mathrm{CAD} \mathrm{a}=p<0.001 \& \mathrm{~b}=p<0.01$; CAD vs normal coronary $\mathrm{c}=p<0.05$

\section{Statistical analysis}

Difference between means was assessed with the students $t$ test. Value of $p$ less than 0.05 was considered statistically significant. The Chi-squared tests as well as Pearson correlation coefficient (r) were also used to evaluate the strength of any association between different variables.

\section{Results}

Basic characteristics of the subjects in the three groups, namely controls, CAD and normal coronary are presented in Table 1. Compared with controls and normal coronary, CAD group had greater proportions of smokers, hypertensives and diabetics. The prevalence of smoking was comparatively lower in normal coronary group than in CAD patients and controls. However, the proportion of subjects with diabetes mellitus, hypertension and overweight were much higher in normal coronary group than in controls and lower than that of CAD group.

Table 2 shows the mean values of serum total cholesterol, triglycerides, LDL-cholesterol and HDL-cholesterol for the three study groups. Compared with controls and normal coronary groups, CAD patients had significantly lower levels of HDL-Cholesterol $(p<0.001)$ and higher levels of triglycerides (control vs CAD; $p<0.001$ ). There was no significant difference between CAD and normal coronary subjects in terms of total cholesterol and LDL-cholesterol. But these levels were significantly lower in CAD when compared to controls $(p<0.05)$. We found that the subjects participated as controls had an increased mean level of total cholesterol $(220 \mathrm{mg} \%)$ than the reference value for total cholesterol $(200 \mathrm{mg} \%)$, may be due to unrestricted dietary habits. Further, the ratio of total cholesterol to HDLCholesterol (a better risk indicator) was found to be higher $(p<0.001)$ in CAD group $(6.2 \pm 2.0)$ than that of controls $(5.3 \pm 1.7)$ and normal coronary group $(5.3 \pm 1.97)$.

Status of PON-1 activity in serum together with the levels of three major antioxidant vitamins-E, $-\mathrm{C}$ and $\beta$-carotene, as well as lipid peroxides are given in Table 3. Mean activity of PON-1 measured, as arylesterase was significantly lower 

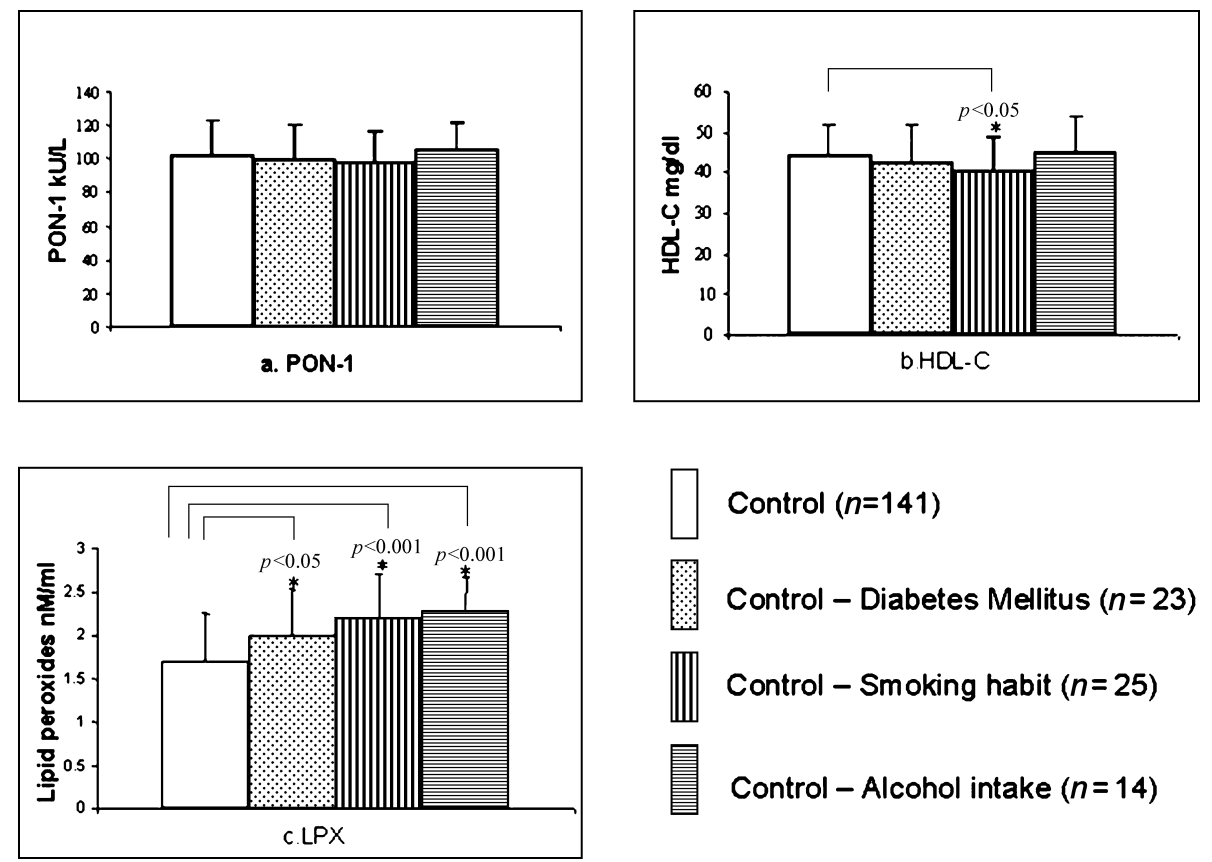

Fig. 1. Influence of risk factors such as diabetes mellitus, smoking habit and alcohol intake on (a) PON-1 activity, (b) HDL-cholesterol and (c) lipid peroxides among control subjects.

in CAD group than that of normal coronary $(p<0.05)$ and control group $(p<0.001)$. On the other hand CAD patients had significantly higher levels of lipid peroxides, an index of oxidative stress compared to normal coronary $(p<0.05)$ and controls $(p<0.001)$. Among the antioxidant vitamins, (except vitamin-E) vitamin-C and $\beta$-carotene levels were significantly lower in patients having CAD than in controls $(p<0.001)$. As in CAD group, these vitamin levels were found to be lower in normal coronary group than that of controls, but the difference was not significant statistically, may be due to smaller sample size in normal coronary group.

The distributions of PON-1 along with lipid peroxides were analyzed in $\mathrm{CAD}$ and non-CAD subjects as a function of the status of diabetes mellitus. There was no significant difference in PON-1 activity between diabetics and nondiabetics among CAD, normal coronary and control groups. However, the levels of lipid peroxides were found enhanced in diabetics compared to non-diabetics in control $\left[\mathrm{DM}^{+}\right.$ $\left.2.02 \pm 0.52(n=35) \mathrm{vs} \mathrm{DM}^{-} 1.80 \pm 0.60(n=212), p<0.05\right]$, normal coronary $\left[\mathrm{DM}^{+} 2.13 \pm 0.56(n=9)\right.$ vs $\mathrm{DM}^{-} 1.72 \pm$ $0.53(n=36), p<0.05]$ and CAD groups $\left[\mathrm{DM}^{+} 2.25 \pm 0.59\right.$ $(n=112)$ vs $\mathrm{DM}^{-} 2.12 \pm 0.63(n=172)$ not significant]. Irrespective of diabetic status CAD patients had remarkably higher levels of lipid peroxides than that of non-diabetic controls.

Data from the control group was further analyzed to see the possible influence of lifestyle factors such as cigarette smoking and alcohol intake as well as diabetes mellitus on PON-1 activity, HDL-cholesterol and lipid peroxide levels.
From the Fig. 1, it was obvious that alcohol intake alone slightly enhanced the activity of PON-1 (did not attain statistical significance) compared to non-users with a concomitant increase in lipid peroxide $(p<0.001)$ and no alteration in HDL-cholesterol level. But, smoking habit seemed to depress the level of HDL-cholesterol $(p<0.05)$ and enhance levels of lipid peroxides $(p<0.001)$. Briefly, alcohol intake, smoking habits and diabetes mellitus among controls adversely influenced the oxidative stress. But, these risk factors did not influence serum PON-1 activity.

In this study population PON-1 activity was found varied from 26 to $170 \mathrm{kU} /$ liter. When the three-study groups were further categorized according to PON-1 activity in tertiles, as lowest [PON <84], middle [PON 84-104] and highest category $[\mathrm{PON} \geq 105$ ], remarkable differences in the distribution of subjects were observed. It was observed that more subjects in the CAD group (45\%) belong to lowest (first) PON-1 tertile. The corresponding proportions in the control (22\%) and normal coronary (22\%) groups were much lower. On the other hand, more subjects both from the control ( second tertile $=35 \% \&$ third tertile $=43 \%$ ) and normal coronary groups (second tertile $=39 \% \&$ third tertile $=39 \%$ ) were found distributed in the middle and highest PON-1 tertiles and it was only $30 \%$ and $25 \%$ respectively in the case of CAD group. These differences are statistically significant $[p<0.01$, Chi-square $]$, and indicating inverse association of PON-1 activity with CAD.

In order to see the influence of biochemical variables on PON-1 activity we have analyzed the association of 

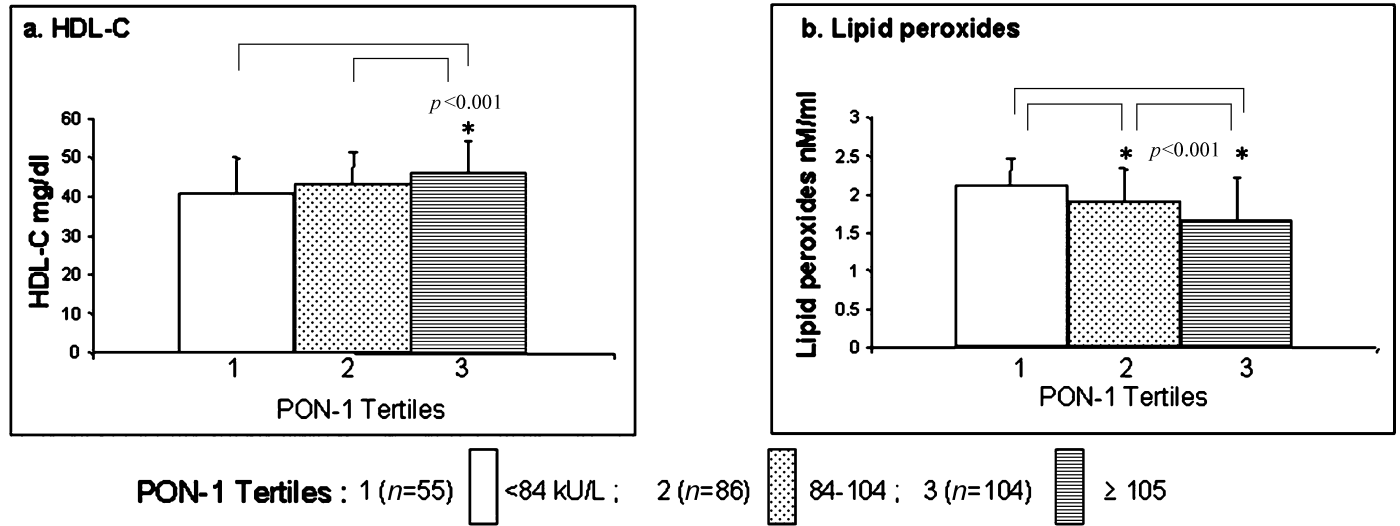

Fig. 2. Influence of biochemical variables such as (a) HDL-cholesterol and (b) lipid peroxides on PON-1 activity in tertiles among control subjects.

HDL-cholesterol, lipid peroxides (Fig. 2), and antioxidant vitamins-E, $-\mathrm{C} \& \beta$-carotene with PON-1 activity in tertiles in control subjects. As expected, the mean levels of HDLcholesterol showed significant positive association and lipid peroxides showed negative association with PON-1 activity in tertiles, although there was no significant correlation (r) between PON-1 and HDL-cholesterol and PON-1 and lipid peroxides. Further, we have also found no association between PON-1 tertile activity and corresponding levels of antioxidant vitamins-E, $-\mathrm{C}$ and $\beta$-carotene (data not shown).

The associations of PON-1 activity and HDL-Cholesterol with the severity of coronary artery disease in terms of number of diseased vessels as one-, two- and three-vessel coronary artery disease based on angiographic reports were further analyzed in patients. All the patients with proven coronary artery disease were found to have reduced PON-1 activity [1-vessel disease $88 \pm 24(n=100)$; 2-vessel disease $89 \pm 25(n=89)$; 3-vessel disease $88 \pm 26(n=95)$ vs normal coronary $96 \pm 22(n=45), p<0.05]]$ and HDLcholesterol [1-vessel disease $32 \pm 8.3$; 2-vessel disease $34 \pm 7.7$; 3-vessel disease $34 \pm 9.6$ vs normal coronary $38 \pm 9.4 ; p<0.001,0.01 \& 0.05$ respectively] compared to that of normal coronary subjects. However we could not observe any difference in these two variables as the disease progressed from one-vessel to two- or three-vessel disease indicating lack of association with the extend of coronary artery disease.

\section{Discussion}

In the present study we have demonstrated that PON-1 activity and HDL-cholesterol are significantly reduced in subjects with angiographilly proven coronary artery disease compared to normal coronary and control subjects. This finding is in agreement with the reports that have found reduced PON-1 activities in CAD [8-10]. This effect was also reported to be independent of PON-1 genotype [9, 22]. PON-1 activity measured using phenyl acetate as substrate has been thought to be more closely reflecting PON-1 concentration and it minimizes the risk of genotype variation. Its activity can be decreased in subjects either due to decreased synthesis or inactivation under oxidative stress by lipid peroxides. In support of this concept we have observed an enhanced oxidative stress in CAD patients as characterized by increased levels of lipid peroxides.

The activity of PON-1 can be influenced by acquired factors such as diet, life style and diseases. Most studies [23, 24], but not others [25], have found that PON-1 activity is reduced in Type- 1 and Type- 2 diabetic patients. In our study there was no significant difference in PON-1 activity between diabetics and non-diabetics with and without CAD. This finding does not support the concept that PON-1 plays an important role in predisposition of subjects to diabetes mellitus. In addition, we could not observe any association between PON-1 and cigarette smoking habits in control subjects, although HDL-cholesterol was found decreased in smokers. To better confirm this finding, future study in a large number of healthy subjects need to be conducted.

It has been suggested that consumption of red wine or flavonoid containing drinks [26] as well as moderate alcohol intake [27] increased serum PON-1 activity. In agreement with this we also found a slight positive association between serum PON-1 activity and alcohol consumption in control subjects. However, the beneficial effect of alcohol intake was not evident in the levels of HDL-cholesterol or lipid peroxides. Surprisingly alcohol intake enhanced the lipid peroxides leading to oxidative stress. So, one possibility for the enhanced PON-1 activity observed in alcohol users might be an adaptive enzyme induction to limit the excess oxidative stress and thus suggesting an important physiological role of PON-1 in host defense.

PON-1, an anti-oxidant enzyme, circulates in blood in 
association with HDL particle and hence HDL is likely to be an important determinant of PON-1 activity. In support of this, we have observed a direct relationship between mean HDL-Cholesterol levels and tertiles of PON-1 activity in controls, although there is no significant correlation (r) between PON-1 and HDL-cholesterol. This can be explained partly by the fact that certain other HDL characteristics such as particle size, surface phospholipids, and apoproteins are most important to hold and stabilize PON-1 in HDL particle.

Oxidants are products of normal metabolism and inflammatory response. Inactivation and removal of these reactive oxygen species depend on reaction involving antioxidant defense systems, which constitute a diverse group of compounds with different properties. In this study, patients with CAD were found to have inadequate antioxidant status as evidenced by lower concentrations of PON-1, vitamin C, and $\beta$-carotene. Lipid peroxides that are substrates as well as inhibitors of PON-1 activity were found enhanced in smokers, alcohol users, diabetics and CAD patients. But we could observe depressed PON-1 activity only in patients with established CAD. This could be a combined effect of inadequate antioxidant capacity, excess oxidative stress and inflammatory response that are prevalent in patients with CAD. The lower activity of PON-1 can depress the ability of circulating HDL particle to protect LDL from oxidation, to participate in reverse cholesterol transport pathway, and to inhibit monocyte-endothelial cell interaction. All these appear to be important in the inflammatory response in artery that promotes atherogenesis. This study indicates that low activity of PON-1 can enhance one's risk of developing atherosclerotic heart disease. Hence preserving or even increasing PON-1 activity by interventional means such as drugs or diet rich in flavonoids and antioxidant vitamins may be beneficial in reducing the risk of CAD.

In conclusion, this study demonstrates that PON-1 activity and HDL-cholesterol are lower in subjects with angiographically documented coronary artery disease than in subjects with normal coronary and healthy volunteers. Further, serum levels of PON-1 and HDL-cholesterol show inverse association with the presence of coronary artery disease but not to the severity of coronary disease in terms of number of diseased vessals.

\section{Acknowledgments}

The authors wish to thank the Director, Sree Chitra Tirunal Institute for Medical Sciences and Technology, Trivandrum, India for providing necessary facilities to carry out the work. The authors would also like to thank Professor J.M. Tharakan, Head of the Dept of Cardiology of this Institute for providing the blood samples and relevant clinical details of the patients.

\section{References}

[1] Ross, R.: Atherosclerosis an inflammatory disease. New Engl. J. Med., 340, 115-118, 1999.

[2] Watson, A.D., Berliner, J.A., Hama, S.Y., La Du, B.N., Fuall, K.F., Fogelman, A.M., and Navab, M.: Protective effect of HDL-paraoxonase: inhibition of the biological activity of minimally oxidized LDL. J. Clin. Invest., 96, 2882-2891, 1996.

[3] Mackness, M.I., Arrol, S., and Durrington, P.N.: Paraoxonase prevents accumulation of lipid peroxides in LDL. FEBS Lett., 286, 152-154, 1991.

[4] Mackness, M.I., Abott, C.A., Arrol, S., and Durrington, P.N.: The role of HDL and lipid soluble antioxidant vitamins in inhibiting LDL oxidation. Biochem. J., 294, 829-835, 1993.

[5] Durrington, P.N., Mackness, B., and Mackness, M.I.: Paraoxonase and atherosclerosis. Arterioscler. Thromb. Vasc. Biol., 21, 473-480, 2001.

[6] Mackness, M.I., Mackness, B., Durrington, P.N., Connelly, P.W., and Hegele, R.A.: Paraoxonase: Biochemistry, genetics and relationship to plasma lipoproteins. Curr. Opin. Lipidol., 7, 69-76, 1996.

[7] Aviram, M., Rosenblat, M., Billecke, S., Erogul, J., Sorenson, R., Bisgaier, C.L., Newton, R.S., and La Du, B.: Human serum paraoxonase is inactivated by oxidized LDL and reserved by antioxidants. Free Radic. Biol. Med., 26, 892904, 1999.

[8] Ayub, A., Mackness, M.I., Arrol, S., Mackness, B., Patel, J., and Durrington, P.N.: Serum paraoxonase after myocardial infarction. Arterioscler. Thromb. Vasc. Biol., 19, 330-335, 1999.

[9] Mackness, B., Davies, G.K., Turkie, W., Lee, E., Roberts, D.H., Hills, E., Roberts, C., Durrington, P.N., and Mackness, M.I.: Paraoxonase status in coronary artery disease: are activity and concentration more important than genotype? Arterioscler. Thromb. Vasc. Biol., 21, 1451-1457, 2001.

[10] Mackness, B., Durrington, P., McElduff, P., John, Y., Naheed, A., Michael, W., and Michael, M.: Low paraoxonase activity predicts coronary events in the Caerphelly prospective study. Circulation, 107, 2775-2779, 2003.

[11] Shih, D.M., Gu, L., Xia, Y.R., Navab, M., Li, W.F., Hama, S., Castellani, L.W., Furlong, C.E., Costa, L.G., Fogelman, A.M., and Lusis, A.J.: Mice lacking serum paraoxonase are susceptible to organophosphate toxicity and atherosclerosis. Nature, 394, 284-287, 1998.

[12] Tward, A., Xia, Y.-R., Wang, X.-P., Shi, Y.-S., Park, C., Castellani, L.W., Lusis, A.J., and Shih, D.M.: Decreased Atherosclerotic Lesion Formation in Human Serum Paraoxonase Transgenic Mice. Circulation, 106, 484-490, 2002.

[13] Aviram, M., Hardak, E., Vaya, J., Mahmood, S., Milo, S., Hoffman, A., Billicke, S., Draganov, D., and Rosenblat, M.: Human serum Paraoxonase Q and R selectively decrease lipid peroxides in human coronary and carotid atherosclerotic lesions: PON-1 esterase and peroxides-like activities. Circulation, 101, 2510-2517, 2000.

[14] Player, J.R., Eza, L.C., Bullen, M.F., and Evans, D.A.P.: Genetic polymorphism and inter-ethnic variability of plasma 
paraoxonase-1 activity. J. Med. Genet., 13, 337-342, 1976.

[15] Jayakumari, N., Ambikakumari, V., Balakrisgnan, K.G., and Iyer, K.S.: Distribution of cholesterol in HDL and its sub fractions in patients with coronary atherosclerotic heart disease. Ind. Heart J., 45, 265-268, 1993.

[16] Jayakumari, N., Ambikakumari, V., Balanrishnan, K.G., and Iyer, K.S.: Antioxidant status in relation to free radical production during stable and unstable anginal syndromes. Atherosclerosis, 94, 183-190, 1992.

[17] Beuge, J.A. and Aust, S.D.: Thiobarbituric acid assay for lipid peroxides. Methods Enzymol., 52, 306-310, 1978.

[18] Desai, D.: Vitamin E analysis, methods for animal tissues. Methods Enzymol., 105, 138-147, 1984.

[19] Omaye, S.T., Turnbull, J.D., and Sauberlich, H.E.: Selected methods for the determination of ascorbic acid in animal cells, tissues and fluids. Methods Enzymol., 62, 9-11, 1979.

[20] McCormick, D.B.: Vitamins: Methods for determination of vitamin A and $\beta$-carotene, in Textbook of Clinical Chemistry, ed. By N.W., Tietz, W.B, Saunders company, West Washington square, Philadelphia, 932-934, 1986.

[21] Lorenz, K., Flatter, B., and Augustine, E.: Aryl esterase in serum: Elaboration and clinical application of fixed incubation method. Clin. Chem., 25, 1714-1720, 1979.

[22] Jarvick, G.P., Hatsukami, T.S., Carlson, C., Richter, R.J., Jampsa, R., Brophy, V.H., Margolin, S., Rieder, M., Nickerson, D., Schellenberg, G.D., Heagerty, P.J., and Furlong, C.E.: Paraoxonase activity, but not haplotype utilizing the linkage disequilibrium structure, predicts vascular disease. Arterioscler. Thromb. Vasc. Biol., 23, 1465-1471, 2003.

[23] Boemi, M., Leviev, I., Sirolla, C., Pieri, C., Marra, M., and James, R.W.: Serum paraoxonase is reduced in type 1 diabetes compared to non-diabetic, first-degree relatives: Influence of the ability of HDL to protect LDL from oxidation. Atherosclerosis, 155, 229-235, 2001.

[24] Agachen, B., Yilmaz, H., Karaali, Z., and Ishir, T.: Paraoxonase 55 and 192 polymorphism and its relationship to serum paraoxonase activity and lipids in urkish patients with non-insulin dependent diabetes mellitus. Cell Biochem. Funct., 22, 163-168, 2004.

[25] Kopprasch, S., Pietzch, J., Kuhlish, E., and Graessier, J.: Lack of association between serum paraoxonase activities and increased oxidation of LDL in impaired glucose tolerance and newly diagnosed diabetes mellitus. J. Clin. Endocrinol. Metab., 88, 1711-1716, 2003.

[26] Aviram, M., Domfeld, L., Rosenblat, M., Volkova, N., Kaplan, M., Coleman, R., Hayek, T., Presser, D., and Fubrman, B.: Pomegranate juice consumption reduces oxidative stress, atherogenic modification of LDL and platelet aggregation: Studies in human and atherosclerotic apo-E deficient mice. Am. J. Clin. Nutr., 71, 1062-1076, 2000.

[27] van der Gaag, M.S., van Tol, A., Scheek, L.M., James, R.W., Urgert, R., Schaafsma, G., and Hendriks, H.F.: Daily moderate alcohol consumption increases serum paraoxonase activity, a diet-controlled, randomized intervention study in middle-aged men. Atherosclerosis, 147, 405-410, 1999. 